TRANSACTIONS OF THE

AMERICAN MATHEMATICAL SOCIETY

Volume 362, Number 12, December 2010, Pages 6279-6291

S 0002-9947(2010)05235-3

Article electronically published on July 14, 2010

\title{
COMPLETELY MULTIPLICATIVE FUNCTIONS TAKING VALUES IN $\{-1,1\}$
}

\author{
PETER BORWEIN, STEPHEN K. K. CHOI, AND MICHAEL COONS
}

Abstract. Define the Liouville function for $A$, a subset of the primes $P$, by $\lambda_{A}(n)=(-1)^{\Omega_{A}(n)}$, where $\Omega_{A}(n)$ is the number of prime factors of $n$ coming from $A$ counting multiplicity. For the traditional Liouville function, $A$ is the set of all primes. Denote

$$
L_{A}(x):=\sum_{n \leq x} \lambda_{A}(n) \quad \text { and } \quad R_{A}:=\lim _{n \rightarrow \infty} \frac{L_{A}(n)}{n} .
$$

It is known that for each $\alpha \in[0,1]$ there is an $A \subset P$ such that $R_{A}=\alpha$. Given certain restrictions on the sifting density of $A$, asymptotic estimates for $\sum_{n \leq x} \lambda_{A}(n)$ can be given. With further restrictions, more can be said. For an odd prime $p$, define the character-like function $\lambda_{p}$ as $\lambda_{p}(p k+i)=(i / p)$ for $i=1, \ldots, p-1$ and $k \geq 0$, and $\lambda_{p}(p)=1$, where $(i / p)$ is the Legendre symbol (for example, $\lambda_{3}$ is defined by $\lambda_{3}(3 k+1)=1, \lambda_{3}(3 k+2)=-1(k \geq 0)$ and $\left.\lambda_{3}(3)=1\right)$. For the partial sums of character-like functions we give exact values and asymptotics; in particular, we prove the following theorem.

Theorem. If $p$ is an odd prime, then

$$
\max _{n \leq x}\left|\sum_{k \leq n} \lambda_{p}(k)\right| \asymp \log x .
$$

This result is related to a question of Erdős concerning the existence of bounds for number-theoretic functions. Within the course of discussion, the ratio $\phi(n) / \sigma(n)$ is considered.

\section{INTRODUCTION}

Let $\Omega(n)$ be the number of distinct prime factors in $n$ (with multiple factors counted multiple times). The Liouville $\lambda$-function is defined by

$$
\lambda(n):=(-1)^{\Omega(n)} .
$$

Therefore, $\lambda(1)=\lambda(4)=\lambda(6)=\lambda(9)=\lambda(10)=1$ and $\lambda(2)=\lambda(5)=\lambda(7)=$ $\lambda(8)=-1$. In particular, $\lambda(p)=-1$ for any prime $p$. It is well known (e.g. see $\S 22.10$ of [10]) that $\Omega$ is completely additive; i.e, $\Omega(m n)=\Omega(m)+\Omega(n)$ for any $m$ and $n$, and hence $\lambda$ is completely multiplicative, i.e., $\lambda(m n)=\lambda(m) \lambda(n)$ for all $m, n \in \mathbb{N}$. It is interesting to note that on the set of square-free positive integers $\lambda(n)=\mu(n)$, where $\mu$ is the Möbius function. In this respect, the Liouville $\lambda_{-}$ function can be thought of as an extension of the Möbius function.

Received by the editors June 13, 2008.

2000 Mathematics Subject Classification. Primary 11N25, 11N37; Secondary 11A15.

Key words and phrases. Liouville lambda function, multiplicative functions.

This research was supported in part by grants from NSERC of Canada and MITACS. 
Similar to the Möbius function, many investigations surrounding the $\lambda$-function concern the summatory function of initial values of $\lambda$; that is, the sum

$$
L(x):=\sum_{n \leq x} \lambda(n) .
$$

Historically, this function has been studied by many mathematicians, including Liouville, Landau, Pólya, and Turán. Recent attention to the summatory function of the Möbius function has been given by $\mathrm{Ng}$ [16, 17]. Larger classes of completely multiplicative functions have been studied by Granville and Soundararajan [7, 8, 9].

One of the most important questions is that of the asymptotic order of $L(x)$. More formally, the question is to determine the smallest value of $\vartheta$ for which

$$
\lim _{x \rightarrow \infty} \frac{L(x)}{x^{\vartheta}}=0 .
$$

It is known that the value of $\vartheta=1$ is equivalent to the prime number theorem [14, 15] and that $\vartheta=\frac{1}{2}+\varepsilon$ for any arbitrarily small positive constant $\varepsilon$ is equivalent to the Riemann hypothesis [3]. (The value of $\frac{1}{2}+\varepsilon$ is best possible, as $\limsup _{x \rightarrow \infty} L(x) / \sqrt{x}>.061867$; see Borwein, Ferguson, and Mossinghoff [4.) Indeed, any result asserting a fixed $\vartheta \in\left(\frac{1}{2}, 1\right)$ would give an expansion of the zero-free region of the Riemann zeta function, $\zeta(s)$, to $\Re(s) \geq \vartheta$.

Unfortunately, a closed form for determining $L(x)$ is unknown. This brings us to the motivating question behind this investigation: are there functions similar to $\lambda$ so that the corresponding summatory function does yield a closed form?

Throughout this investigation $P$ will denote the set of all primes. As an analogue to the traditional $\lambda$ and $\Omega$, define the Liouville function for $A \subset P$ by

$$
\lambda_{A}(n)=(-1)^{\Omega_{A}(n)},
$$

where $\Omega_{A}(n)$ is the number of prime factors of $n$ coming from $A$ counting multiplicity. Alternatively, one can define $\lambda_{A}$ as the completely multiplicative function with $\lambda_{A}(p)=-1$ for each prime $p \in A$ and $\lambda_{A}(p)=1$ for all $p \notin A$. Every completely multiplicative function taking only \pm 1 values is built this way. The class of functions from $\mathbb{N}$ to $\{-1,1\}$ is denoted $\mathcal{F}(\{-1,1\}$ ) (as in [8]). Also, define

$$
L_{A}:=\sum_{n \leq x} \lambda_{A}(n) \quad \text { and } \quad R_{A}:=\lim _{n \rightarrow \infty} \frac{L_{A}(n)}{n} .
$$

In this paper, we first consider questions regarding the properties of the function $\lambda_{A}$ by studying the function $R_{A}$. The rest of this paper considers an extended investigation of those functions in $\mathcal{F}(\{-1,1\})$ which are character-like in nature (meaning that they agree with a real Dirichlet character $\chi$ at non-zero values). While these functions are not really direct analogues of $\lambda$, much can be said about them. Indeed, we can give exact formulae and sharp bounds for their partial sums. Within the course of discussion, the ratio $\phi(n) / \sigma(n)$ is considered.

\section{Properties of $L_{A}(x)$}

Define the generalized Liouville sequence as

$$
\mathfrak{L}_{A}:=\left\{\lambda_{A}(1), \lambda_{A}(2), \ldots\right\} .
$$

Theorem 1. The sequence $\mathfrak{L}_{A}$ is not eventually periodic. 
Proof. Towards a contradiction, suppose that $\mathfrak{L}_{A}$ is eventually periodic; say the sequence is periodic after the $M$-th term and has period $k$. Now there is an $N \in \mathbb{N}$ such that for all $n \geq N$, we have $n k>M$. Since $A \neq \varnothing$, pick $p \in A$. Then

$$
\lambda_{A}(p n k)=\lambda_{A}(p) \cdot \lambda_{A}(n k)=-\lambda_{A}(n k) .
$$

But $p n k \equiv n k(\bmod k)$, a contradiction to the eventual $k$-periodicity of $\mathfrak{L}_{A}$.

Corollary 1. If $A \subset P$ is non-empty, then $\lambda_{A}$ is not a Dirichlet character.

Proof. This is a direct consequence of the non-periodicity of $\mathfrak{L}_{A}$.

To get more acquainted with the sequence $\mathfrak{L}_{A}$, we study the partial sums $L_{A}(x)$ of $\mathfrak{L}_{A}$, and to study these, we consider the Dirichlet series with coefficients $\lambda_{A}(n)$.

Starting with singleton sets $\{p\}$ of the primes, a nice relation becomes apparent; for $\Re(s)>1$

$$
\frac{\left(1-p^{-s}\right)}{\left(1+p^{-s}\right)} \zeta(s)=\sum_{n=1}^{\infty} \frac{\lambda_{\{p\}}(n)}{n^{s}}
$$

and for sets $\{p, q\}$,

$$
\frac{\left(1-p^{-s}\right)\left(1-q^{-s}\right)}{\left(1+p^{-s}\right)\left(1+q^{-s}\right)} \zeta(s)=\sum_{n=1}^{\infty} \frac{\lambda_{\{p, q\}}(n)}{n^{s}} .
$$

For any subset $A$ of primes, since $\lambda_{A}$ is completely multiplicative, for $\Re(s)>1$ we have

$$
\begin{aligned}
\mathcal{L}_{A}(s) & :=\sum_{n=1}^{\infty} \frac{\lambda_{A}(n)}{n^{s}}=\prod_{p}\left(\sum_{l=0}^{\infty} \frac{\lambda_{A}\left(p^{l}\right)}{p^{l s}}\right) \\
& =\prod_{p \in A}\left(\sum_{l=0}^{\infty} \frac{(-1)^{l}}{p^{l s}}\right) \prod_{p \notin A}\left(\sum_{l=0}^{\infty} \frac{1}{p^{l s}}\right)=\prod_{p \in A}\left(\frac{1}{1+\frac{1}{p^{s}}}\right) \prod_{p \notin A}\left(\frac{1}{1-\frac{1}{p^{s}}}\right) \\
& =\zeta(s) \prod_{p \in A}\left(\frac{1-p^{-s}}{1+p^{-s}}\right) .
\end{aligned}
$$

This relation leads us to our next theorem, but first let us recall a vital piece of notation from the Introduction.

Definition 1. For $A \subset P$ denote

$$
R_{A}:=\lim _{n \rightarrow \infty} \frac{\lambda_{A}(1)+\lambda_{A}(2)+\ldots+\lambda_{A}(n)}{n} .
$$

The existence of the limit $R_{A}$ is guaranteed by Wirsing's Theorem. In fact, Wirsing in 20] showed more generally that every real multiplicative function $f$ with $|f(n)| \leq 1$ has a mean value; i.e., the limit

$$
\lim _{x \rightarrow \infty} \frac{1}{x} \sum_{n \leq x} f(n)
$$

exists. Furthermore, in 19 Wintner showed that

$$
\lim _{x \rightarrow \infty} \frac{1}{x} \sum_{n \leq x} f(n)=\prod_{p}\left(1+\frac{f(p)}{p}+\frac{f\left(p^{2}\right)}{p^{2}}+\ldots\right)\left(1-\frac{1}{p}\right) \neq 0
$$

if and only if $\sum_{p}|1-f(p)| / p$ converges; otherwise the mean value is zero. This gives the following theorem. 
Theorem 2. For the completely multiplicative function $\lambda_{A}(n)$, the limit $R_{A}$ exists and

$$
R_{A}= \begin{cases}\prod_{p \in A} \frac{p-1}{p+1} & \text { if } \sum_{p \in A} p^{-1}<\infty \\ 0 & \text { otherwise. }\end{cases}
$$

Example 1. For any prime $p, R_{\{p\}}=\frac{p-1}{p+1}$.

To be a little more descriptive, let us make some notational comments. Denote by $\mathcal{P}(P)$ the power set of the set of primes. Note that

$$
\frac{p-1}{p+1}=1-\frac{2}{p+1} \text {. }
$$

Recall from above that $R: \mathcal{P}(P) \rightarrow \mathbb{R}$ is defined by

$$
R_{A}:=\prod_{p \in A}\left(1-\frac{2}{p+1}\right) \text {. }
$$

It is immediate that $R$ is bounded above by 1 and below by 0 , so that we need only consider that $R: \mathcal{P}(P) \rightarrow[0,1]$. It is also immediate that $R_{\varnothing}=1$ and $R_{P}=0$.

Remark 1 . For an example of a subset of primes with mean value in $(0,1)$, consider the set $K$ of primes defined by

$$
K:=\left\{p_{n} \in P: p_{n}=\min _{q>n^{3}}\{q \in P\} \text { for } n \in \mathbb{N}\right\} .
$$

Since there is always a prime in the interval $\left(x, x+x^{5 / 8}\right]$ (see Ingham [12]), these primes are well defined; that is, $p_{n+1}>p_{n}$ for all $n \in \mathbb{N}$. The first few values give

$$
K=\{11,29,67,127,223,347,521,733,1009,1361, \ldots\} .
$$

Note that

$$
\frac{p_{n}-1}{p_{n}+1}>\frac{n^{3}-1}{n^{3}+1},
$$

so that

$$
R_{K}=\prod_{p \in K}\left(\frac{p-1}{p+1}\right)>\prod_{n=2}^{\infty}\left(\frac{n^{3}-1}{n^{3}+1}\right)=\frac{2}{3} .
$$

Also $R_{K}<(11-1) /(11+1)=5 / 6$, so that

and $R_{K} \in(0,1)$.

$$
\frac{2}{3}<R_{K}<\frac{5}{6}
$$

There are some very interesting and important examples of sets of primes $A$ for which $R_{A}=0$. Indeed, results of von Mangoldt [18, and Landau [14, 15, give the following equivalence.

Theorem 3. The prime number theorem is equivalent to $R_{P}=0$.

We may be a bit more specific regarding the values of $R_{A}$ for $A \in \mathcal{P}(P)$. For each $\alpha \in(0,1)$, there is a set of primes $A$ such that

$$
R_{A}=\prod_{p \in A}\left(\frac{p-1}{p+1}\right)=\alpha .
$$

This result is a special case of some general theorems of Granville and Soundararajan 8 . 
Theorem 4 (Granville and Soundararajan [8]). The function $R: \mathcal{P}(P) \rightarrow[0,1]$ is surjective. That is, for each $\alpha \in[0,1]$ there is a set of primes $A$ such that $R_{A}=\alpha$.

Proof. This follows from Corollary 2 and Theorem 4 (ii) of [8] with $S=\{-1,1\}$.

In fact, let $S$ denote a subset of the unit disk and let $\mathcal{F}(S)$ be the class of totally multiplicative functions such that $f(p) \in S$ for all primes $p$. Granville and Soundararajan 8 prove very general results concerning both the Euler product spectrum $\Gamma_{\theta}(S)$ and the spectrum $\Gamma(S)$ of the class $\mathcal{F}(S)$.

The following theorem gives asymptotic formulas for the mean value of $\lambda_{A}$ if a certain condition on the sifting density of $A$ in $P$ is assumed.

Theorem 5. Suppose $A$ is a subset of primes with sifting density $\frac{1-\kappa}{2}$, where $-1 \leq \kappa \leq 1$. More precisely,

$$
\sum_{\substack{p \leq x \\ p \in A}} \frac{\log p}{p}=\frac{1-\kappa}{2} \log x+O(1)
$$

and $-1 \leq \kappa \leq 1$.

If $0<\kappa \leq 1$, then

$$
\sum_{n \leq x} \lambda_{A}(n)=(1+o(1)) c_{\kappa} \kappa x(\log x)^{\kappa-1}
$$

where $c_{\kappa}=\frac{1}{\Gamma(\kappa+1)} \prod_{p}\left(1-\frac{1}{p}\right)^{\kappa}\left(1-\frac{\lambda_{A}(p)}{p}\right)^{-1} ;$ in particular,

$$
R_{A}=\lim _{x \rightarrow \infty} \frac{1}{x} \sum_{n \leq x} \lambda_{A}(n)= \begin{cases}c_{1}=\prod_{p \in A}\left(\frac{p-1}{p+1}\right) & \text { if } \kappa=1, \\ 0 & \text { if } 0<\kappa<1 .\end{cases}
$$

If $-1 \leq \kappa \leq 0$, then

$$
R_{A}=\lim _{x \rightarrow \infty} \frac{1}{x} \sum_{n \leq x} \lambda_{A}(n)=0 .
$$

Proof. This theorem follows from Wirsing's Theorem (Theorem 1.1 on page 27 of [13]) and a generalization of the Wiener-Ikehara Theorem (Theorem 7.7 of [2]).

Recall that Theorem 4 tells us that any $\alpha \in[0,1]$ is a mean value of a function in $\mathcal{F}(\{-1,1\})$. The functions in $\mathcal{F}(\{-1,1\})$ can be put into two natural classes: those with mean value 0 and those with positive mean value.

Asymptotically, those functions with mean value zero are more interesting, and it is in this class that the Liouville $\lambda$-function resides, and in that which concerns the prime number theorem and the Riemann hypothesis. We consider as an extended example a special class of functions with mean value 0 in Section 4 Before this consideration, we ask some questions about those functions $f \in \mathcal{F}(\{-1,1\}$,$) with$ positive mean value.

\section{ONE QUESTION TWICE}

It is obvious that if $\alpha \notin \mathbb{Q}$, then $R_{A} \neq \alpha$ for any finite set $A \subset P$. We also know that if $A \subset P$ is finite, then $R_{A} \in \mathbb{Q}$.

Question 1. Is there a converse to this; that is, for $\alpha \in \mathbb{Q}$ is there a finite subset $A$ of $P$, such that $R_{A}=\alpha$ ? 
The above question can be posed in a more interesting fashion. Indeed, note that for any finite set of primes $A$ we have that

$$
R_{A}=\prod_{p \in A} \frac{p-1}{p+1}=\prod_{p \in A} \frac{\phi(p)}{\sigma(p)}=\frac{\phi(z)}{\sigma(z)},
$$

where $z=\prod_{p \in A} p, \phi$ is Euler's totient function and $\sigma$ is the sum-of-divisors function. Alternatively, we may view the finite set of primes $A$ as determined by the square-free integer $z$. In fact, the function $f$ from the set of square-free integers to the set of finite subsets of primes, defined by

$$
f(z)=f\left(p_{1} p_{2} \cdots p_{r}\right)=\left\{p_{1}, p_{2}, \ldots, p_{r}\right\}, \quad\left(z=p_{1} p_{2} \cdots p_{r}\right),
$$

is bijective, giving a one-to-one correspondence between these two sets.

In this terminology, we ask the question as:

Question 2. Is the image of $\phi(z) / \sigma(z):\{$ square-free integers $\} \rightarrow \mathbb{Q} \cap(0,1)$ a surjection?

That is, for every rational $q \in(0,1)$, is there a square-free integer $z$ such that $\frac{\phi(z)}{\sigma(z)}=q$ ? As a start, Theorem 4 gives a nice corollary.

Corollary 2. If $S$ is the set of square-free integers, then

$$
\left\{x \in \mathbb{R}: x=\lim _{\substack{k \rightarrow \infty \\\left(n_{k}\right) \subset S}} \frac{\phi\left(n_{k}\right)}{\sigma\left(n_{k}\right)}\right\}=[0,1] ;
$$

that is, the set $\{\phi(s) / \sigma(s): s \in S\}$ is dense in $[0,1]$.

Proof. Let $\alpha \in[0,1]$ and $A$ be a subset of primes for which $R_{A}=\alpha$. If $A$ is finite we are done, so suppose $A$ is infinite. Write

$$
A=\left\{a_{1}, a_{2}, a_{3}, \ldots\right\},
$$

where $a_{i}<a_{i+1}$ for $i=1,2,3, \ldots$, and define $n_{k}=\prod_{i=1}^{k} a_{i}$. The sequence $\left(n_{k}\right)$ satisfies the needed limit.

\section{The Functions $\lambda_{p}(n)$}

We now turn our attention to a class of those functions in $\mathcal{F}(\{-1,1\})$ with mean value 0 . In particular, we wish to examine functions for which a sort of Riemann hypothesis holds: functions for which $\mathcal{L}_{A}(s)=\sum_{n \in \mathbb{N}} \frac{\lambda_{A}(n)}{n^{s}}$ has a large zero-free region; that is, functions for which $\sum_{n \leq x} \lambda_{A}(n)$ grows slowly. Indeed, the functions we consider in the following sections have partial sums which grow extremely slow, in fact, logarithmically. Because of this slow growth, they give rise to Dirichlet series which converge in the entire right half-plane $\Re(s)>0$. Thus our investigation diverges significantly from functions which are very similar to $\lambda$, but focuses on those that yield some very interesting results.

To this end, let $p$ be a prime number. Recall that the Legendre symbol modulo $p$ is defined as

$$
\left(\frac{q}{p}\right)= \begin{cases}1 & \text { if } q \text { is a quadratic residue modulo } p \\ -1 & \text { if } q \text { is a quadratic non-residue modulo } p \\ 0 & \text { if } q \equiv 0 \quad(\bmod p)\end{cases}
$$


Here $q$ is a quadratic residue modulo $p$ provided $q \equiv x^{2}(\bmod p)$ for some $x \not \equiv 0$ $(\bmod p)$.

Define the function $\Omega_{p}(n)$ to be the number of prime factors, $q$, of $n$ with $\left(\frac{q}{p}\right)=$ -1 ; that is,

$$
\Omega_{p}(n)=\#\left\{q: q \text { is a prime, } q \mid n \text {, and }\left(\frac{q}{p}\right)=-1\right\} .
$$

Definition 2. The modified Liouville function for quadratic non-residues modulo $p$ is defined as

$$
\lambda_{p}(n):=(-1)^{\Omega_{p}(n)} .
$$

Analogous to $\Omega(n)$, since $\Omega_{p}(n)$ counts primes with multiplicities, $\Omega_{p}(n)$ is completely additive, and so $\lambda_{p}(n)$ is completely multiplicative. This being the case, we may define $\lambda_{p}(n)$ uniquely by its values at primes.

Lemma 1. The function $\lambda_{p}(n)$ is the unique completely multiplicative function defined by $\lambda_{p}(p)=1$, and for primes $q \neq p$ by

$$
\lambda_{p}(q)=\left(\frac{q}{p}\right) .
$$

Proof. Let $q$ be a prime with $q \mid n$. Now $\Omega_{p}(q)=0$ or 1 depending on whether $\left(\frac{q}{p}\right)=1$ or -1 , respectively. If $\left(\frac{q}{p}\right)=1$, then $\Omega_{p}(q)=0$, and so $\lambda_{p}(q)=1$.

On the other hand, if $\left(\frac{q}{p}\right)=-1$, then $\Omega_{p}(q)=1$, and so $\lambda_{p}(q)=-1$. In either case, we have 1

$$
\lambda_{p}(q)=\left(\frac{q}{p}\right)
$$

Hence if $n=p^{k} m$ with $p \nmid m$, then we have

$$
\lambda_{p}\left(p^{k} m\right)=\left(\frac{m}{p}\right) .
$$

Similarly, we may define the function $\Omega_{p}^{\prime}(n)$ to be the number of prime factors $q$ of $n$ with $\left(\frac{q}{p}\right)=1$; that is,

$$
\Omega_{p}^{\prime}(n)=\#\left\{q: q \text { is a prime, } q \mid n \text {, and }\left(\frac{q}{p}\right)=1\right\} .
$$

Analogous to Lemma 1 we have the following lemma for $\lambda_{p}^{\prime}(n)$ and theorem relating these two functions to the traditional Liouville $\lambda$-function.

Lemma 2. The function $\lambda_{p}^{\prime}(n)$ is the unique completely multiplicative function defined by $\lambda_{p}^{\prime}(p)=1$ and for primes $q \neq p$ as

$$
\lambda_{p}^{\prime}(q)=-\left(\frac{q}{p}\right) .
$$

Theorem 6. If $\lambda(n)$ is the standard Liouville $\lambda$-function, then

$$
\lambda(n)=(-1)^{k} \cdot \lambda_{p}(n) \cdot \lambda_{p}^{\prime}(n),
$$

where $p^{k} \| n$, i.e., $p^{k} \mid n$ and $p^{k+1} \nmid n$.

\footnotetext{
${ }^{1}$ Note that by using the given definition $\lambda_{p}(p)=\left(\frac{p}{p}\right)=1$.
} 
Proof. It is clear that the theorem is true for $n=1$. Since all functions involved are completely multiplicative, it suffices to show the equivalence for all primes. Note that $\lambda(q)=-1$ for any prime $q$. Now if $n=p$, then $k=1$ and

$$
(-1)^{1} \cdot \lambda_{p}(p) \cdot \lambda_{p}^{\prime}(p)=(-1) \cdot(1) \cdot(1)=-1=\lambda(p) .
$$

If $n=q \neq p$, then

$$
(-1)^{0} \cdot \lambda_{p}(q) \cdot \lambda_{p}^{\prime}(q)=\left(\frac{q}{p}\right) \cdot\left(-\left(\frac{q}{p}\right)\right)=-\left(\frac{q^{2}}{p}\right)=-1=\lambda(q),
$$

and so the theorem is proved.

To mirror the relationship between $L$ and $\lambda$, denote by $L_{p}(n)$ the summatory function of $\lambda_{p}(n)$; that is, define

$$
L_{p}(n):=\sum_{k=1}^{n} \lambda_{p}(k)
$$

It is quite immediate that $L_{p}(n)$ is not positive $2^{2}$ for all $n$ and $p$. To find an example we need only look at the first few primes. For $p=5$ and $n=3$, we have

$$
L_{5}(3)=\lambda_{5}(1)+\lambda_{5}(2)+\lambda_{5}(3)=1-1-1=-1<0 .
$$

Indeed, the next few theorems are sufficient to show that there is a positive proportion (at least $1 / 2$ ) of the primes for which $L_{p}(n)<0$ for some $n \in \mathbb{N}$.

Theorem 7. Let

$$
n=a_{0}+a_{1} p+a_{2} p^{2}+\ldots+a_{k} p^{k}
$$

be the base $p$ expansion of $n$, where $a_{j} \in\{0,1,2, \ldots, p-1\}$. Then we have

$$
L_{p}(n):=\sum_{l=1}^{n} \lambda_{p}(l)=\sum_{l=1}^{a_{0}} \lambda_{p}(l)+\sum_{l=1}^{a_{1}} \lambda_{p}(l)+\ldots+\sum_{l=1}^{a_{k}} \lambda_{p}(l) .
$$

Here the sum over $l$ is regarded as empty if $a_{j}=0$.

Instead of giving a proof of Theorem 7 in this specific form, we will prove a more general result for which Theorem 7 is a direct corollary. To this end, let $\chi$ be a non-principal Dirichlet character modulo $p$ and for any prime $q$ let

$$
f(q):= \begin{cases}1 & \text { if } p=q \\ \chi(q) & \text { if } p \neq q .\end{cases}
$$

We extend $f$ to be a completely multiplicative function and get

$$
f\left(p^{l} m\right)=\chi(m)
$$

for $l \geq 0$ and $p \nmid m$.

Theorem 8. Let $N(n, l)$ be the number of digits $l$ in the base $p$ expansion of $n$. Then

$$
\sum_{j=1}^{n} f(j)=\sum_{l=0}^{p-1} N(n, l)\left(\sum_{m \leq l} \chi(m)\right) .
$$

\footnotetext{
${ }^{2}$ For the traditional $L(n)$, it was conjectured by Pólya that $L(n) \geq 0$ for all $n$, though this was proven to be a non-trivial statement and ultimately false (see Haselgrove [1]).
} 
Proof. We write the base $p$ expansion of $n$ as

$$
n=a_{0}+a_{1} p+a_{2} p^{2}+\ldots+a_{k} p^{k}
$$

where $0 \leq a_{j} \leq p-1$. We then observe that, by writing $j=p^{l} m$ with $p \nmid m$,

$$
\sum_{j=1}^{n} f(j)=\sum_{l=0}^{k} \sum_{\substack{j=1 \\ p^{l} \| j}}^{n} f(j)=\sum_{l=0}^{k} \sum_{\substack{m \leq n / p^{l} \\(m, p)=1}} f\left(p^{l} m\right)
$$

For simplicity, we write

$$
A:=a_{0}+a_{1} p+\ldots+a_{l} p^{l} \quad \text { and } \quad B:=a_{l+1}+a_{l+2} p+\ldots+a_{k} p^{k-l-1}
$$

so that $n=A+B p^{l+1}$ in (9). It now follows from (8) and (9) that

$$
\sum_{j=1}^{n} f(j)=\sum_{l=0}^{k} \sum_{\substack{m \leq n / p^{l} \\(m, p)=1}} \chi(m)=\sum_{l=0}^{k} \sum_{m \leq A / p^{l}+B p} \chi(m)=\sum_{l=0}^{k} \sum_{m \leq A / p^{l}} \chi(m)
$$

because $\chi(p)=0$ and $\sum_{m=a+1}^{a+p} \chi(m)=0$ for any $a$. Now since

$$
a_{l} \leq A / p^{l}=\left(a_{0}+a_{1} p+\ldots+a_{l} p^{l}\right) / p^{l}<a_{l}+1
$$

we have

$$
\sum_{j=1}^{n} f(j)=\sum_{l=0}^{k} \sum_{m \leq a_{l}} \chi(m)=\sum_{l=0}^{p-1} N(n, l)\left(\sum_{m \leq l} \chi(m)\right) .
$$

This proves the theorem.

In this language, Theorem 7 can be stated as follows.

Corollary 3. If $N(n, l)$ is the number of digits $l$ in the base $p$ expansion of $n$, then

$$
L_{p}(n)=\sum_{j=1}^{n} \lambda_{p}(j)=\sum_{l=0}^{p-1} N(n, l)\left(\sum_{m \leq l}\left(\frac{m}{p}\right)\right) .
$$

As an application of this theorem consider $p=3$.

Application 1. The value of $L_{3}(n)$ is equal to the number of 1's in the base 3 expansion of $n$.

Proof. Since $\left(\frac{1}{3}\right)=1$ and $\left(\frac{1}{3}\right)+\left(\frac{2}{3}\right)=0$, if $n=a_{0}+a_{1} 3+a_{2} 3^{2}+\ldots+a_{k} 3^{k}$ is the base 3 expansion of $n$, then the right-hand side of (6) (or equivalently, the right-hand side of (10) $)$ is equal to $N(n, 1)$. The result then follows from Theorem 7 (or equivalently Corollary 3 ).

Note that $L_{3}(n)=k$ for the first time when $n=3^{0}+3^{1}+3^{2}+\ldots+3^{k}$ and is never negative. This is in stark contrast to the traditional $L(n)$, which is negative more often than not. Indeed, we may classify all $p$ for which $L_{p}(n) \geq 0$ for all $n \in \mathbb{N}$.

Theorem 9. The function $L_{p}(n) \geq 0$ for all $n$ exactly for those odd primes $p$ for which

$$
\left(\frac{1}{p}\right)+\left(\frac{2}{p}\right)+\ldots+\left(\frac{k}{p}\right) \geq 0
$$

for all $1 \leq k \leq p$. 
Proof. We first observe from (5) that if $0 \leq r<p$, then

$$
\sum_{l=1}^{r} \lambda_{p}(l)=\sum_{l=1}^{r}\left(\frac{l}{p}\right) .
$$

From Theorem 7 .

$$
\begin{aligned}
\sum_{l=1}^{n} \lambda_{p}(l) & =\sum_{l=1}^{a_{0}} \lambda_{p}(l)+\sum_{l=1}^{a_{1}} \lambda_{p}(l)+\ldots+\sum_{l=1}^{a_{k}} \lambda_{p}(l) \\
& =\sum_{l=1}^{a_{0}}\left(\frac{l}{p}\right)+\sum_{l=1}^{a_{1}}\left(\frac{l}{p}\right)+\ldots+\sum_{l=1}^{a_{k}}\left(\frac{l}{p}\right),
\end{aligned}
$$

because all $a_{j}$ are between 0 and $p-1$. The result then follows.

Corollary 4. For $n \in \mathbb{N}$, we have

$$
0 \leq L_{3}(n) \leq\left[\log _{3} n\right]+1 .
$$

Proof. This follows from Theorem 9. Application 1, and the fact that the number of 1's in the base three expansion of $n$ is $\leq\left[\log _{3} n\right]+1$.

As a further example, let $p=5$.

Corollary 5. The value of $L_{5}(n)$ is equal to the number of 1 's in the base 5 expansion of $n$ minus the number of 3 's in the base 5 expansion of $n$. Also for $n \geq 1$,

$$
\left|L_{5}(n)\right| \leq\left[\log _{5} n\right]+1 \text {. }
$$

Recall from above that $L_{3}(n)$ is always non-negative but $L_{5}(n)$ isn't. Also, $L_{5}(n)=k$ for the first time when $n=5^{0}+5^{1}+5^{2}+\ldots+5^{k}$, and $L_{5}(n)=-k$ for the first time when $n=3 \cdot 5^{0}+3 \cdot 5^{1}+3 \cdot 5^{2}+\ldots+3 \cdot 5^{k}$.

Remark 2. The reason for specific $p$ values in the preceding two corollaries is that, in general, it's not always the case that $\left|L_{p}(n)\right| \leq\left[\log _{p} n\right]+1$.

We now return to our classification of primes for which $L_{p}(n) \geq 0$ for all $n \geq 1$.

Definition 3. Denote by $\mathcal{L}^{+}$the set of primes $p$ for which $L_{p}(n) \geq 0$ for all $n \in \mathbb{N}$.

We have found, by computation, that the first few values in $\mathcal{L}^{+}$are

$$
\mathcal{L}^{+}=\{3,7,11,23,31,47,59,71,79,83,103,131,151,167,191,199,239,251, \ldots\} .
$$

By inspection, $\mathcal{L}^{+}$doesn't seem to contain any primes $p$, with $p \equiv 1(\bmod 4)$. This is not a coincidence, as demonstrated by the following theorem.

Theorem 10. If $p \in \mathcal{L}^{+}$, then $p \equiv 3(\bmod 4)$.

Proof. Note that if $p \equiv 1(\bmod 4)$, then

$$
\left(\frac{a}{p}\right)=\left(\frac{-a}{p}\right)
$$

for all $1 \leq a \leq p-1$, so that

$$
\sum_{a=1}^{\frac{p-1}{2}}\left(\frac{a}{p}\right)=0
$$


Consider the case that $\left(\frac{(p-1) / 2}{p}\right)=1$. Then

$$
\sum_{a=1}^{\frac{p-1}{2}}\left(\frac{a}{p}\right)=\sum_{a=1}^{\frac{p-1}{2}-1}\left(\frac{a}{p}\right)+\left(\frac{(p-1) / 2}{p}\right)=\sum_{a=1}^{\frac{p-1}{2}-1}\left(\frac{a}{p}\right)+1
$$

so that

$$
\sum_{a=1}^{\frac{p-1}{2}-1}\left(\frac{a}{p}\right)=-1<0
$$
have

On the other hand, if $\left(\frac{(p-1) / 2}{p}\right)=-1$, then since $\left(\frac{(p-1) / 2}{p}\right)=\left(\frac{(p-1) / 2+1}{p}\right)$, we

$$
\sum_{a=1}^{\frac{p-1}{2}}\left(\frac{a}{p}\right)=\sum_{a=1}^{\frac{p-1}{2}+1}\left(\frac{a}{p}\right)-\left(\frac{(p-1) / 2+1}{p}\right)=\sum_{a=1}^{\frac{p-1}{2}+1}\left(\frac{a}{p}\right)+1
$$

so that

$$
\sum_{a=1}^{\frac{p-1}{2}+1}\left(\frac{a}{p}\right)=-1<0
$$

\section{A BOUND FOR $\left|L_{p}(n)\right|$}

Above we were able to give exact bounds on the function $\left|L_{p}(n)\right|$. As explained in Remark 2, this is not always possible, though an asymptotic bound is easily attained with a few preliminary results.

Lemma 3. For all $r, n \in \mathbb{N}$ we have $L_{p}\left(p^{r} n\right)=L_{p}(n)$.

Proof. For $i=1, \ldots, p-1$ and $k \in \mathbb{N}, \lambda_{p}(k p+i)=\lambda_{p}(i)$. This relation immediately gives for $k \in \mathbb{N}$ that $L_{p}(p(k+1)-1)-L_{p}(p k)=0$, since $L_{p}(p-1)=0$. Thus

$$
L_{p}\left(p^{r} n\right)=\sum_{k=1}^{p^{r} n} \lambda_{p}(k)=\sum_{k=1}^{p^{r-1} n} \lambda_{p}(p k)=\sum_{k=1}^{p^{r-1} n} \lambda_{p}(p) \lambda_{p}(k)=\sum_{k=1}^{p^{r-1} n} \lambda_{p}(k)=L_{p}\left(p^{r-1} n\right) .
$$

The lemma follows immediately.

Theorem 11. The maximum value of $\left|L_{p}(n)\right|$ for $n<p^{i}$ occurs at $n=k \cdot \sigma\left(p^{i-1}\right)$ with value

$$
\max _{n<p^{i}}\left|L_{p}(n)\right|=i \cdot \max _{n<p}\left|L_{p}(n)\right|,
$$

where $\sigma(n)$ is the sum of the divisors of $n$.

Proof. This follows directly from Lemma 3.

Corollary 6. If $p$ is an odd prime, then $\left|L_{p}(n)\right| \ll \log n$; furthermore,

$$
\max _{n \leq x}\left|L_{p}(n)\right| \asymp \log x
$$




\section{Concluding Remarks}

Throughout this paper, we were interested in estimates concerning the partial sums of multiplicative functions in $\mathcal{F}(\{-1,1\})$. An important question is: what can be said about the growth of $\left|\sum_{n \leq x} f(n)\right|$ for any function $f \in \mathcal{F}(\{-1,1\})$ ? This question goes back to Erdős. Indeed, Erdős [5] states: Finally, I would like to mention an old conjecture of mine: let $f(n)= \pm 1$ be an arbitrary number-theoretic function [not necessarily multiplicative]. Is it true that to every $c$ there is a $d$ and an $m$ so that

$$
\left|\sum_{k=1}^{m} f(k d)\right|>c ?
$$

I have made no progress with this conjecture.

Concerning this conjecture, he adds in [6] that the best we could hope for is that

$$
\max _{m d \leq n}\left|\sum_{k=1}^{m} f(k d)\right|>c \log n .
$$

We remark that these questions can also be asked for functions $f(n)$ which take kth roots of unity as values rather than just \pm 1 . However, very little is yet known for this case.

If we restrict Erdös' question to the class of completely multiplicative functions, it may be possible to provide an answer. This would be interesting. Recall that for $p$ an odd prime and $\lambda_{p}$ a character-like function, we have

$$
\max _{N \leq x}\left|\sum_{n \leq N} \lambda_{p}(n)\right| \asymp \log x,
$$

so that the class of character-like functions satisfies Erdős' conjecture.

These functions are part of a larger class of functions called automatic functions (see [1]). Let $\mathbf{T}=(t(n))_{n \geq 1}$ be a sequence with values from a finite set. Define the $k$-kernel of $\mathbf{T}$ as the set

$$
\mathbf{T}^{(k)}=\left\{\left(t\left(k^{l} n+r\right)\right)_{n \geq 0}: l \geq 0 \text { and } 0 \leq r<k^{l}\right\} .
$$

Given $k \geq 2$, we say a sequence $\mathbf{T}$ is $k$-automatic if and only if the $k$-kernel of $\mathbf{T}$ is finite.

As a generalization of our result concerning character-like functions, we ask the following question.

Question 3. Let $f \in \mathcal{F}(\{-1,1\})$ be $k$-automatic for some $k \geq 2$, and suppose that $\sum_{n \leq x} f(n)=o(x)$. Then is it true that $\max _{N \leq x}\left|\sum_{n \leq N} f(n)\right| \asymp \log x$ ?

\section{REFERENCES}

1. J.-P. Allouche and J. Shallit, Automatic sequences, Cambridge University Press, Cambridge, 2003. MR.1997038 (2004k:11028)

2. Paul T. Bateman and Harold G. Diamond, Analytic number theory, World Scientific Publishing Co. Pte. Ltd., Hackensack, NJ, 2004. An introductory course. MR2111739 (2005h:11208)

3. P. Borwein, S. Choi, B. Rooney, and A. Weirathmuller, The Riemann Hypothesis: A Resource for the Afficionado and Virtuoso Alike, CMS Books in Mathematics, vol. 27, Springer, New York, 2008. MR2463715 (2009k:11002)

4. P. Borwein, R. Ferguson, and M.J. Mossinghoff, Sign changes in sums of the Liouville function, Math. Comp. 77 (2008), no. 263, 1681-1694. MR2398787 (2009b:11227) 
5. P. Erdős, Problems and results on combinatorial number theory. In A survey of combinatorial theory (Proc. Internat. Sympos., Colorado State Univ., Fort Collins, Colo., 1971), pages 117-138. North-Holland, Amsterdam, 1973. MR0360509 (50:12957)

6. P. Erdős and R. L. Graham, Old and new problems and results in combinatorial number theory, volume 28 of Monographies de L'Enseignement Mathématique [Monographs of L'Enseignement Mathématique]. Université de Genève L'Enseignement Mathématique, Geneva, 1980. MR.592420 (82j:10001)

7. Andrew Granville and K. Soundararajan, Motivating the multiplicative spectrum, Topics in number theory (University Park, PA, 1997), Math. Appl., vol. 467, Kluwer Acad. Publ., Dordrecht, 1999, pp. 1-15. MR.1691308 (2000m:11088)

8. Andrew Granville and K. Soundararajan, The spectrum of multiplicative functions, Ann. of Math. (2) 153 (2001), no. 2, 407-470. MR.1829755 (2002g:11127)

9. Andrew Granville and K. Soundararajan, Decay of mean values of multiplicative functions, Canad. J. Math. 55 (2003), no. 6, 1191-1230. MR2016245 (2005b:11157)

10. G. H. Hardy and E. M. Wright, An Introduction to the Theory of Numbers, fifth ed., The Clarendon Press Oxford University Press, New York, 1979. MR.568909 (81i:10002)

11. C. B. Haselgrove, A disproof of a conjecture of Pólya, Mathematika 5 (1958), 141-145. MR.0104638 (21:3391)

12. A. E. Ingham, On the difference between consecutive primes, Quart. J. Math. Oxford 8 (1937), $255-266$.

13. Henryk Iwaniec and Emmanuel Kowalski, Analytic number theory, American Mathematical Society Colloquium Publications, vol. 53, American Mathematical Society, Providence, RI, 2004. MR2061214 (2005h:11005)

14. Edmund Landau, Neuer Beweis der Gleichung $\sum \frac{\mu(k)}{k}=0$, Inaugural-Dissertation, Berlin, 1899.

15. Edmund Landau, Über die Äquivalenz zweier Hauptsätze der analytischen Zahlentheorie, Wien. Sitz. 120 (1911), 973-988.

16. Nathan $\mathrm{Ng}$, The distribution factor of values of the summatory function of the Möbius function, Notes of the Canad. Math. Soc. 34 (2002), no. 5, 5-8.

17. Nathan Ng, The distribution of the summatory function of the Möbius function, Proc. London Math. Soc. (3) 89 (2004), no. 2, 361-389. MR2078705 (2005f:11215)

18. Hans Carl Friedrich von Mangoldt, Beweis der Gleichung $\sum_{k=0}^{\infty} \frac{\mu(k)}{k}=0$, Proc. Royal Pruss. Acad. of Sci. of Berlin (1897), 835-852.

19. Aurel Wintner, The Theory of Measure in Arithmetical Semi-Groups, publisher unknown, Baltimore, Md., 1944. MR0015083(7:367a)

20. E. Wirsing, Das asymptotische Verhalten von Summen über multiplikative Funktionen. II, Acta Math. Acad. Sci. Hungar. 18 (1967), 411-467. MR0223318 (36:6366)

Department of Mathematics, Simon Fraser University, Burnaby, British Columbia, CANADA V5A 1S6

E-mail address: pborwein@cecm.sfu.ca

Department of Mathematics, Simon Fraser University, Burnaby, British Columbia, CANADA V5A 1 S6

E-mail address: kkchoi@math.sfu.ca

The Fields Institute, 222 College Street, Toronto, Ontario, Canada M5T 3J1

E-mail address: mcoons@math.uwaterloo.ca 